\title{
Dual oxidase 1 and NADPH oxidase 2 exert favorable effects in cervical cancer patients by activating immune response
}

Sang Yeon $\mathrm{Cho}^{1 \dagger}$, Sungha Kim ${ }^{2+}$, Mi-Ju Son², Gwanghun Kim³ ${ }^{3}$ Parul Singh ${ }^{4}$, Ha Neul Kim ${ }^{5}$, Hei-Gwon Choi ${ }^{6}$, Heon Jong Yoo ${ }^{7,8}$, Young Bok Ko ${ }^{7,8}$, Byung Seok Lee ${ }^{9,10^{*}}$ and Hyuk Soo Eun ${ }^{9,10^{*}}$ (i)

\begin{abstract}
Background: Nicotinamide adenine dinucleotide phosphate (NADPH) oxidase-derived reactive oxygen species (ROS) not only can promote cancer progression, but also they have recently emerged as mediators of the mucosal immune system. However, the roles and clinical relevance of the collective or individual NADPH oxidase (NOX) family genes in cervical cancer have not been studied.

Methods: We investigated the clinical significance of the NOX family genes using data from 307 patients with cervical cancer obtained from The Cancer Genome Atlas. Bioinformatics and experimental analyses were performed to examine NOX family genes in cervical cancer patients.

Results: Dual Oxidase1 (DUOX1) and Dual Oxidase 2 (DUOX2) mRNA levels were upregulated 57.9- and 67.5-fold, respectively, in cervical cancer patients. The protein expression of DUOX1, DUOX2, and NOX2 also identified in cervical squamous cell carcinoma tissues. Especially, DUOX1 and DUOX2 mRNA levels were significantly increased in patients infected with human papillomavirus (HPV) 16. Moreover, high DUOX1 mRNA levels were significantly associated with both favorable overall survival and disease-free survival in cervical cancer patients. High NOX2 mRNA levels was significantly associated with favorable overall survival. Gene set enrichment analyses revealed that high DUOX1 and NOX2 expression was significantly correlated with the enrichment of immune pathways related to interferon (IFN)-alpha, IFN-gamma, and natural killer (NK) cell signaling. Cell-type identification by estimating relative subsets of known RNA transcript analyses indicated that the fraction of innate immune cells, including NK cells, monocytes, dendritic cells, and mast cells, was elevated in patients with high DUOX1 expression.
\end{abstract}

Conclusions: DUOX1 and NOX2 expression are associated with mucosal immunity activated in cervical squamous cell carcinoma and predicts a favorable prognosis in cervical cancer patients.

Keywords: NADPH oxidases, Dual oxidases, Uterine cervical neoplasms, Papillomaviridae, Survival, Disease-free survival

\section{Background}

Human papillomavirus (HPV) is the primary etiologic agent of cervical cancer [1]. However, HPV alone is not sufficient for tumor progression; the clinical manifestation of HPV infection depends on the immune response of the host [2]. Tumors are recognized by the

\footnotetext{
* Correspondence: gie001@cnuh.co.kr; hyuksoo@cnuh.co.kr

† Sang Yeon Cho and Sungha Kim contributed equally to this work.

${ }^{9}$ Department of Internal Medicine, Chungnam National University Hospital,

282 Munwha-ro, Jung-gu, Daejeon, Republic of Korea

Full list of author information is available at the end of the article
}

immune system and their development can be stopped or controlled through a process known as immunosurveillance [3]. The mucosal epithelium represents the first line of defense against virus invasion. An immature or weakened innate immunity of the uterine cervical epithelium may exacerbate viral infection. Therefore, despite the improvements in vaccines against HPV, more studies are needed to identify new therapeutic inducers for the reinforcement of the innate immune responses against HPV infection in cervical cancer patients.

(c) The Author(s). 2019 Open Access This article is distributed under the terms of the Creative Commons Attribution 4.0 International License (http://creativecommons.org/licenses/by/4.0/), which permits unrestricted use, distribution, and reproduction in any medium, provided you give appropriate credit to the original author(s) and the source, provide a link to the Creative Commons license, and indicate if changes were made. The Creative Commons Public Domain Dedication waiver (http://creativecommons.org/publicdomain/zero/1.0/) applies to the data made available in this article, unless otherwise stated. 
The NADPH oxidase (NOX) family, the major family of enzymes that catalyze reactive oxygen species (ROS) production, comprises seven members: NOX1-5, dual oxidase (DUOX) 1, and DUOX2 [4]. ROS induce oxidative stress and diverse inflammatory responses [5]. Excessive ROS production by NOX homologs as a result of chronic inflammation can also promote proliferative and invasive malignancies [6]. However, oxidative innate immune defense mechanism mediated by NADPH oxidase family members has been emerged, especially, DUOX plays an important role in host mucosal immunity by producing hydrogen peroxide [7-9]. Host-defense properties of DUOX have also been identified in nonmammalian organisms [10-13]. Homologs of DUOX are found in nearly all multicellular organisms, and DUOX enzymes seem to be evolved to fundamentally serve host immune defense [14]. DUOX1 and DUOX2 may have unique roles in specific arms of the innate immune response. Nevertheless, the immunologic effect of DUOX in the uterine cervical mucosa, which provides the first line of defense to HPV invasion, especially in cervical cancer, has not yet been investigated.

The present study aimed to investigate whether NOX family members are involved in cervical cancer progression or host immunity in response to cervical cancer. We used data from 307 cervical cancer patients obtained from The Cancer Genome Atlas (TCGA). Indeed, we discovered a prognostic value of DUOX1 and NOX2 expression in cervical cancer patients, and we attempted to elucidate the underlying mechanisms by using bioinformatics analyses, including gene set enrichment analysis (GSEA) and cell-type identification by estimating relative subsets of known RNA transcript (CIBERSORT). Moreover, we analyzed the protein expression of NOX2, DUOX1, and DUOX2 using clinical tissue samples from cervical cancer patients.

\section{Methods}

Gene and protein expression profiles

RNAseqV2-RSEM_genes and clinical data from 307 Cervical Squamous Cell Carcinoma and Endocervical Adenocarcinoma (CESC) samples and 3 normal control samples were obtained from The Cancer Genome Atlas (http://portal.gdc.cancer.gov/) and Firebrowse (http:// firebrowse.org/) for gene expression analysis. The validation set (GSE75132) of 30 samples with persistent HPV 16 infection and 11 normal control samples was downloaded from the Gene Expression Omnibus (GEO) database (https://www.ncbi.nlm.nih.gov/geo/). Raw data were initially processed in R v.3.2.5 (http://www.r-project.org). Chip data were normalized with RankNormalize in GenePattern (http://www.broadinstitute.org/cancer/software/ genepattern/). Gene Expression Profiling Interactive Analysis (GEPIA; http://gepia.cancer-pku.cn/) was utilized to compare mRNA expression between cervical cancer patients based on data from TCGA database (https://portal.gdc.cancer.gov/) and 13 normal controls based on data from The Genotype-Tissue Expression (GTEx) Project from the Broad Institute of MIT and Harvard (www.gtexportal.org). Human normal tissue distribution of DUOX1, DUOX2, and NOX2 was analyzed based on RNAseq data extracted from the GTEx project. Protein expression and immunohistochemical (IHC) staining data were obtained from the Human Protein Atlas (HPA) (http://www.proteinatlas.org).

\section{Western blotting}

Total protein samples were isolated from frozen liver tissue using RIPA lysis buffer, containing protease and phosphatase inhibitor cocktail (TransLab, \#3004CLI19SSH). Samples were separated in a 10\% SDSpolyacrylamide gel electrophoresis and transferred onto nitrocellulose membrane (GE Healthcare Life Sciences, \#10600023). After the membranes were blocked in 5\% skim milk for $1 \mathrm{~h}$ at room temperature, they were incubated with primary antibodies overnight at $4{ }^{\circ} \mathrm{C}$ and then with the corresponding secondary antibodies for $1 \mathrm{~h}$ at room temperature. All of the primary antibodies gp91-phox antibody (Santa Cruz Biotechnology, \#K0817) and $\beta$-actin (Cell Signaling, $\# 4970$ s) were used at a dilution of 1:1000 except DUOX1 (Santa Cruz Biotechnology, \#B2817) (1:500) and DUOX2 (Santa Cruz Biotechnology, \#D0317) (1: 500). Secondary antibodies were used at 1:2500 dilution. Immunoreactive bands were detected using the enhanced chemiluminescence (ECL) detection system with a PhosphorImager (GE Healthcare). Protein expression levels were normalized to the levels of the $\beta$ actin, which was used as a loading control.

\section{Patients samples}

Frozen cervical cancer tissue samples were obtained from some of patients with cervical cancer and their controls were obtained from the cohort of the Department of Obstetrics and Gynecology, Chungnam National University Hospital (Daejeon, South Korea) and were analyzed by western blot. In this study, each three normal cervical cancer tissues, early-stage cervical squamous cell carcinoma, advanced-stage cervical squamous cell carcinoma, and endocervical adenocarcinoma tissues deposited with the Human Resources Bank of Korea in Chungnam National University Hospital were used for this study. Authorization for the use of these tissues for research purposes and ethical approval were obtained from the Institutional Review Board of Chungnam National University Hospital (IRB number: 2019-05-087). Written informed consents, which were approved by 
Institutional Review Board of Chungnam National University Hospital, were received from the entire patients who had provided the tissue.

\section{Functional enrichment analysis}

Gene Set Enrichment Analysis (GSEA) was used to assess enrichment of mRNAs associated with Hallmark and Kyoto Encyclopedia of Genes and Genomes (KEGG) pathways sets [15]. GSEA was conducted using the 10\% of CESC samples with the most strongly upregulated DUOX1 and NOX2 expression and the 10\% of samples with the most strongly downregulated DUOX1 and NOX2 expression. Enrichment maps were visualized in Cytoscape v.3.5.1 (www.cytoscape.org). A $p$-value of less than 0.05 was considered significant.

\section{Analysis of immune cell subsets from mRNA expression profiles}

To quantify the relative abundances of 22 tumorassociated leukocyte subsets in samples from HPVpositive and -negative CESC patients, we utilized the Cell type Identification By Estimating Relative Subsets Of known RNA Transcript (CIBERSORT) method and the LM22 gene signature, which allow for highly sensitive and specific discrimination of hematopoietic cells and were well-designed and validated based on gene expression profiles from Affymetrix Human Genome U133A/Plus2 [16]. CIBERSORT analysis was conducted using the $10 \%$ samples with the most strongly upregulated DUOX1 and NOX2 expression and the $10 \%$ of samples with the most strongly downregulated DUOX1 and NOX2 expression.

\section{Survival analysis}

Survival analysis of cervical cancer patients was performed using GEPIA. The cumulative event (death) rate was calculated by the Kaplan-Meier method, using the time from the date of operation to the date of death as the outcome variable. Survival curves stratified by risk factors were compared by log-rank test, with $p$-values less than 0.05 considered to indicate statistical significance. The median group cutoff was median.

\section{Statistical analysis}

Data were analyzed in Prism version 5.0 (GraphPad Prism Software, La Jolla, CA, USA) and Statistical Package for Social Sciences for Windows version 13.0 (SPSS, Chicago, IL, USA). Distributions between two groups were compared by $t$-test (or by Kolmogorov-Smirnov test when the expected frequency in any group was less than 5) for continuous variables, and by Chi-square test (or Fisher's exact test when the expected frequency in any group was less than 5) for categorical variables. Three or more groups were compared by one-way analysis of variance. A $p$-value of less than 0.05 was considered significant.

\section{Results \\ DUOX1 and DUOX2 are predominantly expressed in cervical cancer patients}

Clinicopathological characteristics of the patients are listed in Table 1. mRNA and protein expression of DUOX and NOX genes was examined in patients with cervical cancer (Fig. 1). DUOX1 and DUOX2 expression was increased by 57.9 - and 67.5-fold, respectively, whereas NOX4 expression was decreased by 0.17 -fold in

Table 1 Clinicopathologic information of the cervical cancer patients

\begin{tabular}{|c|c|}
\hline Feature & Total (\%) \\
\hline Number & $307(100)$ \\
\hline \multicolumn{2}{|l|}{ Age } \\
\hline$\leq 50$ years & $188(61.2)$ \\
\hline$>50$ years & 119 (38.8) \\
\hline \multicolumn{2}{|l|}{ Histological type } \\
\hline Squamous cell carcinoma & $254(82.7)$ \\
\hline Endocervical adenocarcinoma & $47(15.3)$ \\
\hline Adenosquamous carcinoma & $6(2.0)$ \\
\hline \multicolumn{2}{|l|}{ Vital status } \\
\hline Alive & $235(76.5)$ \\
\hline Dead & $72(23.5)$ \\
\hline \multicolumn{2}{|l|}{ Postoperative Treatment } \\
\hline Yes & $103(33.6)$ \\
\hline No & $77(25.1)$ \\
\hline \multicolumn{2}{|l|}{ Clinical stage } \\
\hline । & $163(53.1)$ \\
\hline$\|$ & $70(22.8)$ \\
\hline III & $46(15.0)$ \\
\hline IV & $21(6.8)$ \\
\hline \multicolumn{2}{|l|}{ Morphological type } \\
\hline Non-keratininzing type & $120(39.1)$ \\
\hline Keratininzing type & $55(17.9)$ \\
\hline \multicolumn{2}{|l|}{ Lymphatic invasion } \\
\hline Absent & $72(23.5)$ \\
\hline Present & 80 26.1) \\
\hline \multicolumn{2}{|l|}{ Human papilloma virus status } \\
\hline Negative & $23(7.5)$ \\
\hline Positive (High risk) & $284(92.5)$ \\
\hline Hpv 16 & $172(56.0)$ \\
\hline Hpv 18 & $39(12.7)$ \\
\hline Hpv 45 & $24(7.8)$ \\
\hline Hpv etc & $47(15.3)$ \\
\hline
\end{tabular}




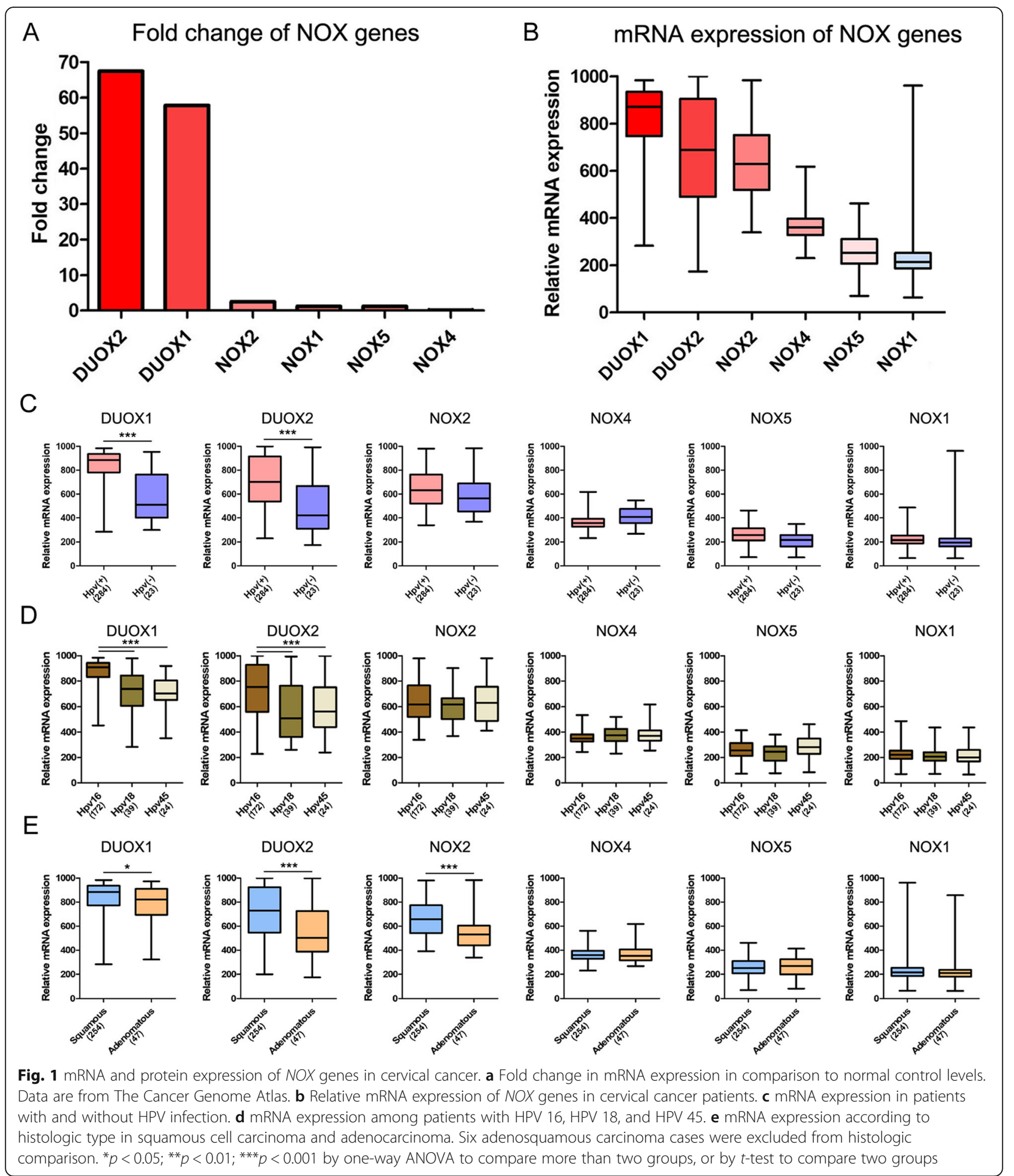

patients compared to normal control subjects (Fig. 1a). DUOX1, DUOX2, and NOX2 protein expression were also identified in our clinical cervical cancer samples (Additional file 1). DUOX1 and DUOX2 were also the most abundant NOX transcripts in cervical cancer patients, whereas NOX3 was the least abundant and was undetectable in normal control subjects (Fig. 1b and Table 2). DUOX and NOX mRNA expression was significantly different according to the presence of HPV infection and histologic type. In cervical cancer patients 
Table 2 Expression of the NADPH oxidase family in patients with cervical cancer

\begin{tabular}{|c|c|c|c|c|}
\hline Symbol & Gene name & Aliases & $\begin{array}{l}\text { Chromosome } \\
\text { location }\end{array}$ & $\begin{array}{l}\text { Log fold } \\
\text { change }\end{array}$ \\
\hline NOX1 & $\begin{array}{l}\text { NADPH } \\
\text { Oxidase } 1\end{array}$ & Mitogenic Oxidase (Pyridine Nucleotide-Dependent Superoxide-Generating) & Xq22.1 & 1.21 \\
\hline NOX2 & $\begin{array}{l}\text { NADPH } \\
\text { Oxidase } 2\end{array}$ & $\begin{array}{l}\text { CYBB (Cytochrome B-245 Beta Chain), Superoxide-Generating NADPH Oxidase Heavy Chain Sub- } \\
\text { unit, Heme-Binding Membrane Glycoprotein Gp91phox, Neutrophil Cytochrome B } 91 \text { KDa } \\
\text { Polypeptide }\end{array}$ & Xp21.1 & 2.50 \\
\hline NOX3 & $\begin{array}{l}\text { NADPH } \\
\text { Oxidase } 3\end{array}$ & Mitogenic Oxidase 2, NADPH Oxidase Catalytic Subunit-Like 3 & $6 q 25.3$ & NA \\
\hline NOX4 & $\begin{array}{l}\text { NADPH } \\
\text { Oxidase } 4\end{array}$ & Kidney Superoxide-Producing NADPH Oxidase, Kidney Oxidase-1 & $11 q 14.3$ & 0.17 \\
\hline NOX5 & $\begin{array}{l}\text { NADPH } \\
\text { Oxidase } 5\end{array}$ & NADPH Oxidase, EF-Hand Calcium Binding Domain 5 & $15 q 23$ & 1.21 \\
\hline DUOX1 & $\begin{array}{l}\text { Dual } \\
\text { Oxidase } 1\end{array}$ & $\begin{array}{l}\text { NADPH Thyroid Oxidase 1, Nicotinamide Adenine Dinucleotide Phosphate Oxidase, Flavoprotein } \\
\text { NADPH Oxidase, Large NOX 1, Long NOX } 1\end{array}$ & $15 q 21.1$ & 57.9 \\
\hline DUOX2 & $\begin{array}{l}\text { Dual } \\
\text { Oxidase } 2\end{array}$ & NADPH Thyroid Oxidase 2, Nicotinamide Adenine Dinucleotide Phosphate Oxidase & $15 q 21.1$ & 67.5 \\
\hline
\end{tabular}

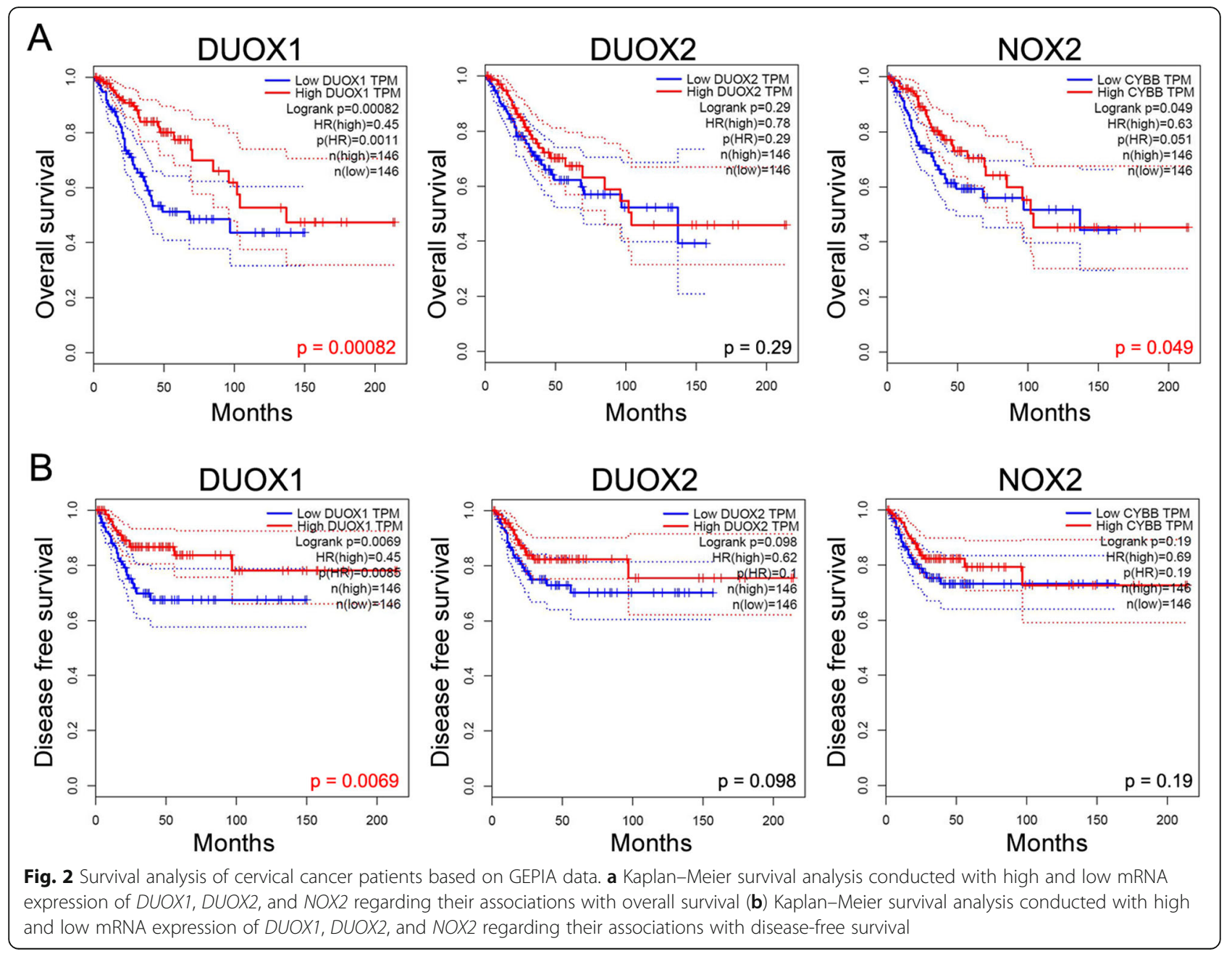


with HPV infection, DUOX1 and DUOX2 mRNA levels were significantly increased as compared to patients without HPV infection (Fig. 1c). DUOX1 and DUOX2 mRNA levels were significantly higher in patients with HPV 16 than in patients with HPV 18 and HPV 45 (Fig. 1d). In addition, mRNA and protein levels of DUOX1 and DUOX2 were higher in patients with cervical squamous cell carcinoma than in those with endocervical adenocarcinoma (Fig. 1e and Additional file 1). However, mRNA levels of NOX family members were not significantly associated with clinical stage and pathologic stage (Additional file 2). Moreover, mRNA expression of $D U O X 1, D U O X 2$, and NOX2 was also significantly increased according to the GEPIA database, as shown in Additional file 3. The normal tissues distribution of human DUOX1, DUOX2, and NOX2 is illustrated in Additional file 4.

\section{Cervical cancer patients with high expression of DUOX1 and NOX2 have a favorable prognosis}

Based on the log-rank test in GEPIA, abundant mRNA expression of DUOX1 (hazard ratio $0.45,95 \%$ confidence interval, $p=0.00082$ ) and NOX2 (hazard ratio 0.63, 95\% confidence interval, $p=0.049)$ was significantly associated with better prognosis of CESC patients in terms of overall survival (Fig. 2a). High mRNA expression of DUOX1 (hazard ratio $0.45,95 \%$ confidence interval, $p=$ $0.0069)$ was significantly associated with better prognosis of CESC patients in disease-free survival (Fig. 2b). In addition, NOX1, NOX4, and NOX5 mRNA levels were not significantly associated with the prognosis of cervical cancer patients.

\section{Immune pathways strongly associated with DUOX1 and NOX2 expression}

Using GSEA and enrichment network visualization, enrichment of mRNAs associated with Hallmark pathways and KEGG pathways (Fig. 3) were investigated in the $10 \%$ CESC samples with the most upregulated DUOX1 and NOX2 expression and in the $10 \%$ of samples with the most downregulated DUOX1 and NOX2 expression. In Hallmarks pathways, high DUOX1 and NOX2 mRNA expression was significantly associated with immune pathways related to interferon (IFN)-alpha and IFNgamma (Fig. 3a and Table 3). The NES (Normalized Enrichment Score) values of IFN-alpha and -gamma responses associated with DUOX1 were 2,17 and 1.85 . The NES values of IFN-gamma, inflammatory response, and IFN-alpha responses related with DUOX2 were 2,93, 2.77 , and 2.69 , respectively.

In KEGG pathways, genes associated with immune pathways, including NK cells, T-cell receptor, B-cell receptor, cytosolic DNA sensing, Toll-like receptor, and retinoic acid-inducible gene-I (RIG-I) receptor were significantly enriched under high DUOX1 mRNA expression. However, repression of DUOX1 mRNA expression significantly enriched for genes related with cancerrelated pathways, including focal adhesion, extracellular matrix receptor interaction, transforming growth factorbeta signaling, and cell adhesion (Fig. 3b). Meanwhile, NOX2 expression enriched for several immune pathways associated with cytokine cytokine-receptor interactions, Janus kinase/signal transducers and activators of transcription (JAK/STAT) signaling, intestinal immunity, Toll-like receptor signaling, RIG-I receptor signaling, cytosolic DNA sensing, T cell receptor, B cell receptor, and NK cell signaling. However, drug-, xenobiotic-, and retinol-metabolic pathways were significantly enriched in samples with downregulated NOX2 mRNA expression (Fig. 3b).

Innate and adaptive immune cell subsets are increased in patients with high DUOX1 and NOX2 expression

CIBERSORT was used to estimate the abundances of immune cell subsets and evaluate the changes in immune cell subsets within tumor micro-environment in cervical cancer (Fig. 4 and Additional files 5 and 6). The analysis was carried out using the $10 \%$ samples with the highest and lowest DUOX1 and NOX2 expression, and revealed a change in abundance in 22 immune cell types (Fig. 4a). Furthermore, the IHC staining of DUOX1 and NOX2 protein was examined in cervical cancer based on data from the Human Protein Atlas (Fig. 4b). It is discovered that the IHC staining of DUOX1 was increased in secretary cells of uterine cervical glands in cervical cancer tissues. The NOX2 was selectively stained intraepithelial infiltrating cells in cervical cancer tissue (Fig. 4b).

Next, we specifically investigated the changes in abundance of adaptive and innate immune cells. Increased abundances of innate immune cells, including NK cells, monocytes, dendritic cells, and mast cells, and decreased abundances of adaptive immune cells, including B cells, $\mathrm{CD} 8 \mathrm{~T}$ cells, and CD4 $\mathrm{T}$ cells, were identified in the patients with high DUOX1 expression compared to the patients with low DUOX1 expression (Fig. 4c). Additionally, in the validation data set, high mRNA levels of DUOX1 were also associated with increased innate immune cells, including NK cells and mast cells, and a decreased fraction of $\mathrm{B}$ cells (Additional file 5). On the other hand, increased percentages of CD8 T cells and follicular helper $\mathrm{T}$ cells and decreased percentages of $\mathrm{B}$ cells and CD4 $\mathrm{T}$ cells in adaptive immune cells were identified in patients with NOX2 high expression (Fig. 4d and Additional file 6). In innate immune cells, the M1/M2 macrophage ratio and neutrophils were increased in patients with high NOX2 expression (Additional file 6). 


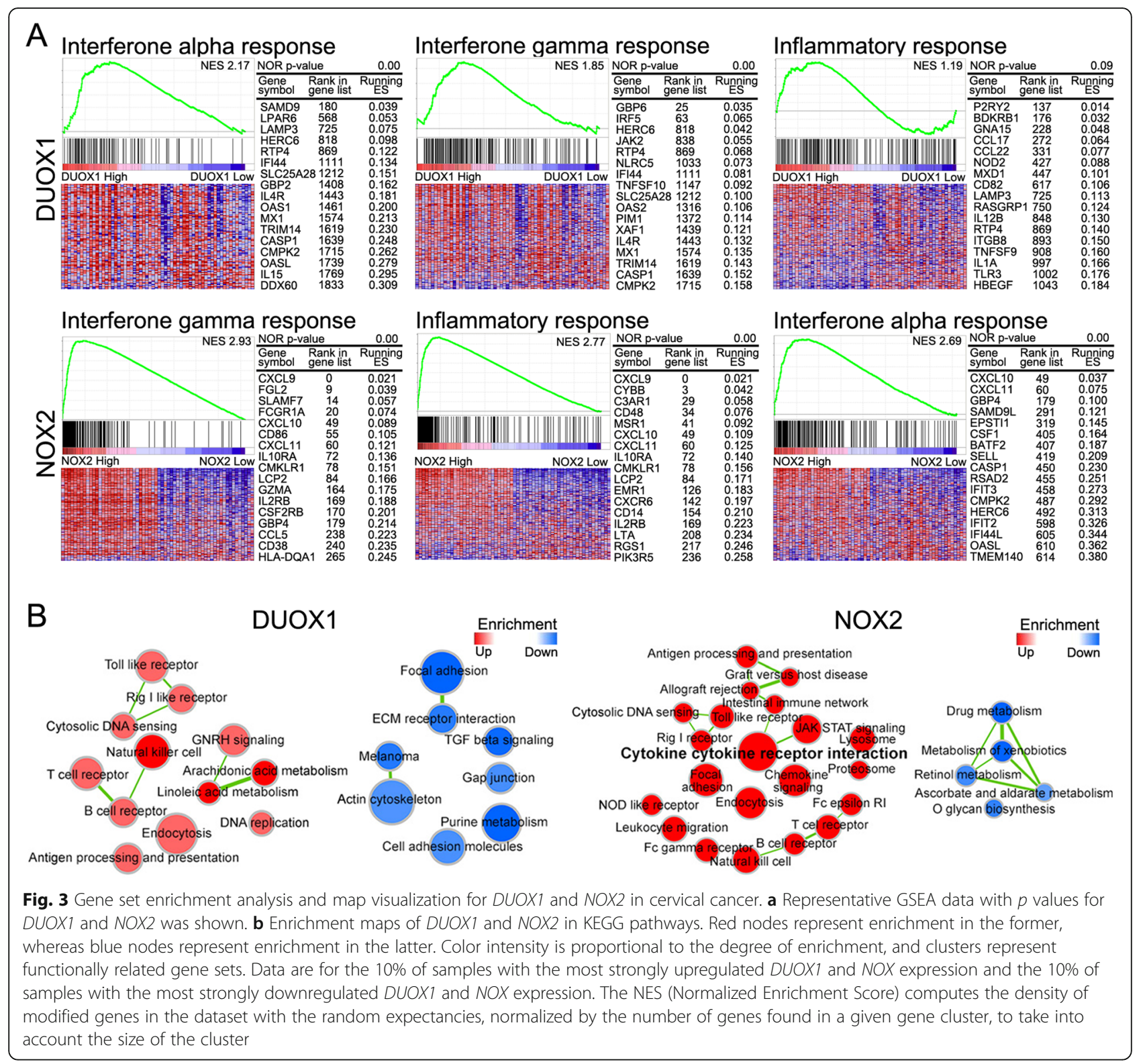

\section{Discussion}

We tried to identify new therapeutic targets for the reinforcement of immune responses against HPV infection. This study was the first to examine the immunologic role and clinical significance of NADPH oxidase family members in cervical cancer patients. We initially evaluated DUOX1 and DUOX2 mRNA levels in the normal ectocervix, endocervix, and vagina (Additional file 4). Interestingly, we found that DUOX1 and DUOX2 mRNA levels were dramatically increased in cervical cancer patients infected with HPV 16 (Fig. 1d). DUOX1 and DUOX2 protein expression were also identified in cervical squamous cell carcinoma (Additional file 1). In line with our findings, a previous study reported that $D U O X$ and $D U O X$-derived ROS were upregulated in the respiratory mucosa upon influenza virus infection [17]. Moreover, in our study, high expression levels of DUOX1 mRNA were significantly associated with favorable overall survival as well as disease-free survival in cervical cancer patients. Indeed, several studies were reported that the relationship between expression and prognostic effect of DUOX1 depend on the cancer tissue type. For example, DUOX enzymes were first identified in thyroid tissues and were found to be involved in thyroid hormone biosynthesis [18-20]. In thyroid cancer, DUOX1 is upregulated upon radiation, and DUOX1dependent $\mathrm{H}_{2} \mathrm{O}_{2}$ production promotes persistent DNA damage and genome instability, which might contribute to cancer development [21, 22]. In contrast, in the respiratory tract, DUOX1 is mostly expressed in the tracheal and bronchial epithelium [9], and DUOX1 mRNA 
Table 3 Hallmark pathways of DUOX1 and NOX2 in cervical cancer

\begin{tabular}{|c|c|c|c|c|}
\hline Term & Size & ES & NES & NOM $p$-val \\
\hline \multicolumn{5}{|l|}{ DUOX1 - Hallmark pathways up } \\
\hline HALLMARK_INTERFERON_ALPHA_RESPONSE & 97 & 0.57 & 2.17 & 0.00 \\
\hline HALLMARK_INTERFERON_GAMMA_RESPONSE & 199 & 0.44 & 1.85 & 0.00 \\
\hline HALLMARK_ESTROGEN_RESPONSE_EARLY & 197 & 0.39 & 1.63 & 0.00 \\
\hline HALLMARK_ESTROGEN_RESPONSE_LATE & 200 & 0.37 & 1.56 & 0.00 \\
\hline HALLMARK_INFLAMMATORY_RESPONSE & 200 & 0.29 & 1.19 & 0.09 \\
\hline HALLMARK_TNFA_SIGNALING_VIA_NFKB & 200 & 0.27 & 1.12 & 0.17 \\
\hline \multicolumn{5}{|l|}{ DUOX1 - Hallmark pathways down } \\
\hline HALLMARK_EPITHELIAL_MESENCHYMAL_TRANSITION & 199 & -0.59 & -2.43 & 0.00 \\
\hline HALLMARK_ANGIOGENESIS & 36 & -0.51 & -1.62 & 0.01 \\
\hline HALLMARK_HEDGEHOG_SIGNALING & 36 & -0.47 & -1.49 & 0.04 \\
\hline HALLMARK_KRAS_SIGNALING_UP & 200 & -0.34 & -1.41 & 0.01 \\
\hline HALLMARK_WNT_BETA_CATENIN_SIGNALING & 42 & -0.39 & -1.29 & 0.11 \\
\hline HALLMARK_APICAL_JUNCTION & 200 & -0.30 & -1.27 & 0.03 \\
\hline \multicolumn{5}{|l|}{ NOX2- Hallmark pathways up } \\
\hline HALLMARK_INTERFERON_GAMMA_RESPONSE & 199 & 0.80 & 2.93 & 0.00 \\
\hline HALLMARK_INFLAMMATORY_RESPONSE & 200 & 0.77 & 2.77 & 0.00 \\
\hline HALLMARK_INTERFERON_ALPHA_RESPONSE & 97 & 0.81 & 2.69 & 0.00 \\
\hline HALLMARK_IL6_JAK_STAT3_SIGNALING & 87 & 0.76 & 2.47 & 0.00 \\
\hline HALLMARK_IL2_STAT5_SIGNALING & 198 & 0.63 & 2.30 & 0.00 \\
\hline HALLMARK_TNFA_SIGNALING_VIA_NFKB & 200 & 0.63 & 2.29 & 0.00 \\
\hline \multicolumn{5}{|l|}{ NOX2- Hallmark pathways down } \\
\hline HALLMARK_GLYCOLYSIS & 199 & -0.36 & -1.50 & 0.00 \\
\hline HALLMARK_NOTCH_SIGNALING & 32 & -0.34 & -1.05 & 0.37 \\
\hline HALLMARK_HEDGEHOG_SIGNALING & 36 & -0.28 & -0.89 & 0.65 \\
\hline HALLMARK_FATTY_ACID_METABOLISM & 156 & -0.22 & -0.88 & 0.80 \\
\hline HALLMARK_PROTEIN_SECRETION & 96 & -0.22 & -0.83 & 0.89 \\
\hline HALLMARK_G2M_CHECKPOINT & 194 & -0.12 & -0.52 & 1.00 \\
\hline
\end{tabular}

and protein are suppressed in lung cancer as a consequence of hypermethylation in the promoter region, and this suppression is associated with poor prognosis [23-25]. Moreover, DUOX1 expression is low in the gastrointestinal tract and has been detected in the stomach lining $[24,26]$. In gastric cancer, mRNA expression of DUOX1 was downregulated, whereas, high levels of DUOX1 mRNA were correlated with poor prognosis, paradoxically [27]. It is conceivable that the expression and prognostic effect of DUOX1 depend on the organ and cancer type.

The role of DUOX2 has been actively investigated in various malignancies $[6,23]$. DUOX2 is the main isoform within the gastrointestinal tract and is expressed most prominently within the colon epithelium and rectal glands $[9,28]$. It has been reported that strong DUOX2 expression accelerates the development of colorectal and pancreatic cancers in patients with inflammatory bowel disease and chronic pancreatitis, respectively [6].
Overexpression of DUOX2/DUOX2A during ulcerative colitis is also thought to be responsible for oxidative DNA damage, which predisposes these patients to colon cancer development [29]. However, in our study, DUOX2 mRNA was detected in the vagina, and rarely detected on the cervix (Additional file 4). DUOX2 mRNA was also dramatically increased in cervical cancer patients; however, high $D U O X 2$ mRNA level was not associated with significant favorable prognosis. Moreover, NOX2 mRNA was rarely detected on the cervix and vagina (Additional file 4). However, NOX2 mRNA was significantly increased in cervical cancer patients with $\mathrm{HPV}$, and high NOX2 mRNA level was significantly associated with favorable overall survival. NOX2 protein expression were also identified in cervical squamous cell carcinoma and adenocarcinoma (Additional file 1). Indeed, it has been indicated that high levels of NOX2 mRNA are implicated in promoting oncogenic 


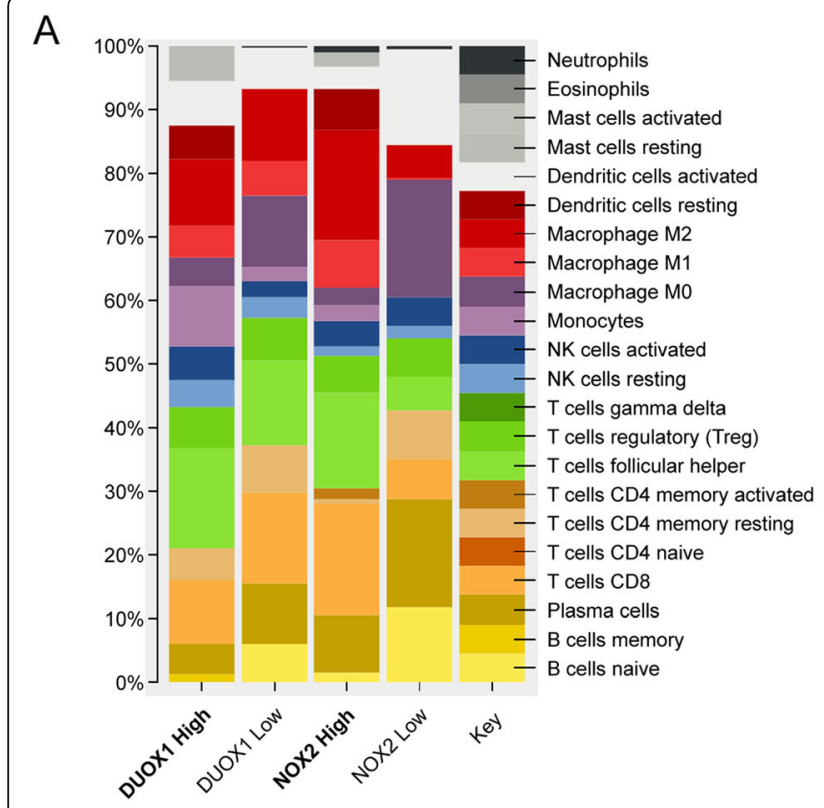

B

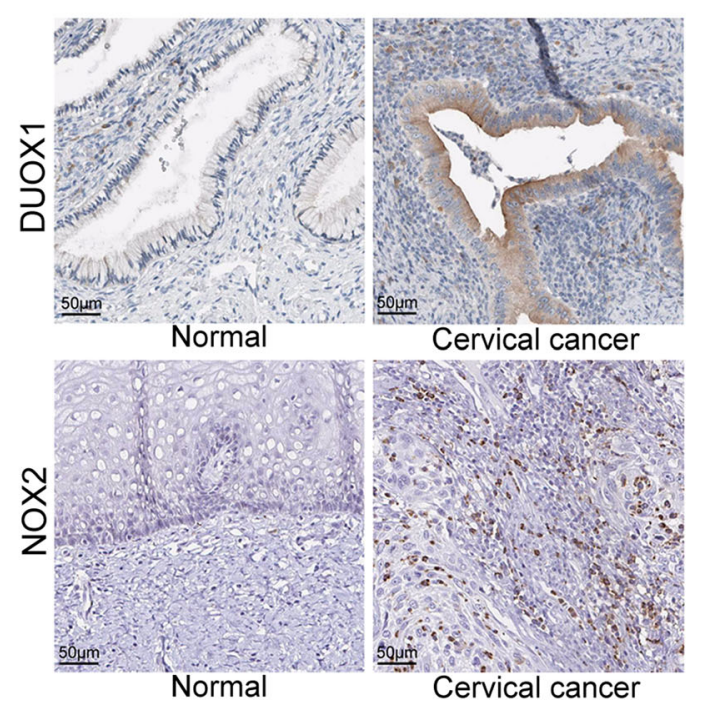

C
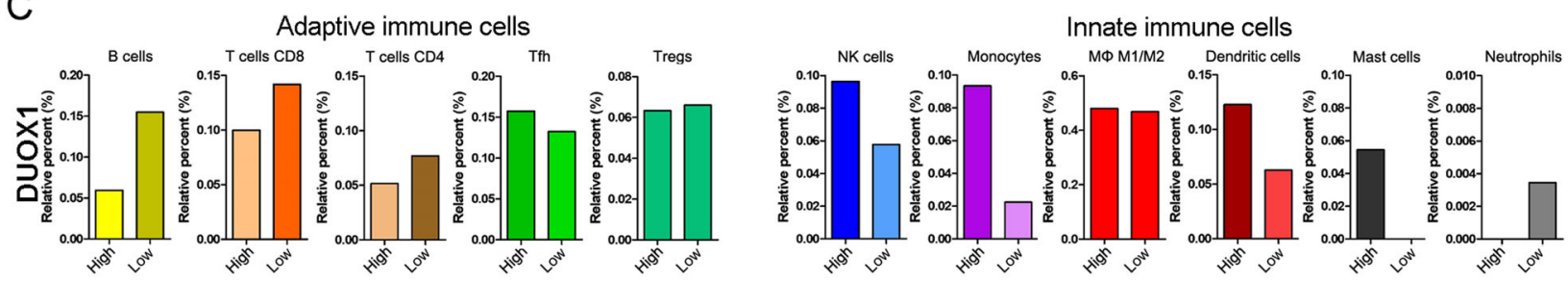

D
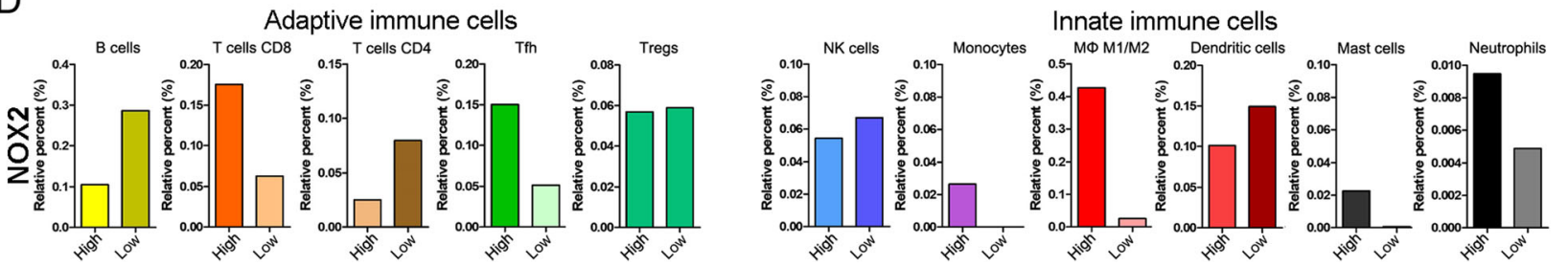

Fig. 4 Immune cell signatures in cervical cancer patients with DUOX1 and NOX2 expression. Estimated mRNA percentages of 22 immune cell subsets (LM22 signature), as calculated by CIBERSORT, in cervical cancer patients with DUOX1 and NOX2 gene expression. a Relative percentages of LM 22 signature subsets in patients with DUOX1 and NOX2 gene expression. b Immunohistochemical staining of DUOX1 and NOX2 adapted from Human Protein Atlas. c Relative percentages of immune cells in patients with high and low DUOX1 mRNA expression. $\mathbf{d}$ Relative percentages of immune cells in patients with high and low NOX2 mRNA expression

characteristics in breast cancer, rectal cancer, and prostate cancer [30-32].

We conducted GSEA to verify the effects of DUOX1 and NOX2 on survival in cervical cancer patients. Notably, expression of both DUOX1 and NOX2 was significantly associated with immune pathways related to IFNalpha and IFN-gamma. IFN is well known to be important for tumor suppression because it not only directly kills tumor cells, but also activates immune cells in the tumor microenvironment [33]. In addition, estrogen response and NK cell signaling pathways were closely related to DUOX1 expression. Moreover, the pathways of TNF alpha and cytokine-cytokine receptor interaction were closely related to NOX2 expression (Table 3 ). The effects of DUOX1 and NOX2 on survival in cervical cancer patients depend commonly on IFN-alpha and IFNgamma, and differential pathways of DUOX1 and NOX2 were identified.

We investigated IHC staining of DUOX1 and NOX2 in cervical cancer tissues based on data from the Human Protein Atlas. Specifically, we discovered that DUOX1 and NOX2 staining in uterine cervical glands and intraepithelial infiltrating cells in cervical cancer tissues. These findings are supported by several recent reports on the presence of DUOX1 in non-epithelial cell types such as T-cells [34], macrophages [35], and innate lymphoid 
cells [36], and the presence of NOX2 in phagocytes [37]. To investigate the immune cell types regulated by DUOX1 and NOX2 mRNA expression in cervical cancer tissues more specifically, we utilized CIBERSORT analysis. Notably, high mRNA levels of DUOX1 were closely related with increased innate immune cells, especially, NK cells, monocytes, dendritic cells, and mast cells, and also with a decreased fraction of adaptive immune cells, including B cells, CD8+ T, and CD4+ T cells. This indicates that DUOX1 expression is highly associated with the innate immune cell response in cervical cancer. Recent evidence indicates that DUOX1 is expressed in innate lymphoid cells, where it has potential roles in innate lymphoid cell polarization, indicating broad host defense functions of DUOX1 [36]. Moreover, the patients with high mRNA expression levels of NOX2 were closely related with increased fractions of M1/M2 macrophages and neutrophils among innate immune cells. In addition, the patients with high mRNA expression levels of NOX2 mRNA levels were related with increased percentages of CD8+ T cells and follicular helper T cells among adaptive immune cells. These findings indicate that NOX2 expression is not only associated with phagocytes, such as macrophages and neutrophils [37], but also with adaptive immune cells, including CD8+ and follicular helper $\mathrm{T}$ cells. Based on GSEA and CIBERSORT analysis, it is suggested that DUOX1 and NOX2 have differential effects on the immune cell-mediated response in cervical cancer patients. In the tumor microenvironment, different types of infiltrating immune cells, including macrophages, dendritic cells, mast cells, NK cells, B cells, and effector $\mathrm{T}$ cells have diverse effects on cancer progression [38]. Especially, NK cells collaborate with dendritic cells to induce an immune response against viral infections and tumors [39]. Activated dendritic cells also play an important role in tumor therapy by acting as natural adjuvants, and tumor-specific follicular helper $\mathrm{T}$ cells act as potent antigen-presenting cells [40,41]. In addition, an increased population of mast cells was related with favorable prognosis [42]. In this study, the increased mRNA levels of DUOX1, $D U O X 2$, and NOX2 in cervical cancer were identified in TCGA and GEO databases. Moreover, the protein expression and their localization of DUOX1 and NOX2 were also confirmed in our own patient samples and Human Protein Atlas database, respectively. However, analyses presented here are mainly suggested on the basis of different databases and there was still a challenge to experimentally validate the proposed underlying mechanism in a large cohort of cervical cancer patients.

\section{Conclusions}

Our results suggest that DUOX1 and NOX2 mediate the IFN-based immune defense against HPV infection, and thereby affect the outcomes of cervical cancer patients. This study has extended our knowledge of the roles of DUOX1 and NOX2 in cervical cancer and shed light on its potential clinical use in cervical cancer patients. The approach of inducing a DUOX1 and NOX2-mediated immune response in uterine cervical mucosa is clinically expected to reinforce immune response to HPV infection and thus increase the survival of cervical cancer patients.

\section{Supplementary information}

Supplementary information accompanies this paper at https://doi.org/10. 1186/s12885-019-6202-3.

Additional file 1. Protein expression of DUOX1, DUOX2, and NOX2 in normal cervix tissues and cervical cancer tissues. (A) Protein expression in normal samples, squamous cell carcinoma and adenocarcinoma. (B) Clinicopathologic information for normal cervix patients and cervical cancer patients.

Additional file 2. NOX family members expression in clinical parameters (A) mRNA expression in three clinical stage. (B) mRNA expression in pathologic stage ( $T$ for tumor size, $N$ for nodal status, and $M$ for status of tumor metastasis).

Additional file 3. NOX family members expression in CESC, based on GEPIA database (Gene Expression Profiling Interactive Analysis).

Additional file 4. Tissue distribution of DUOX1, DUOX2, and NOX2 expression. RNAseq data were extracted from public data deposited by the Broad Institute of MIT and Harvard in the Gene Tissue Expression (GTEx) project.

Additional file 5. mRNA expression and Immune cell signatures in the validation data set (GSE75132). (A) mRNA expression of DUOX2 and NOX2 in patients with HPV 16 infection and normal control samples. (B) Relative percentages of LM 22 signature subsets in patients with DUOX1 gene expression. (C) Relative percentages of immune cells in patients with high and low DUOX1 gene expression. (D) Estimated percentage values of LM22 signature subsets, as calculated by CIBERSORT.

Additional file 6. Estimated percentage values of 22 immune cell signature (LM22 signature) subsets, as calculated by CIBERSORT, between CESC patient groups in cervical cancer patients with DUOX1 and NOX2 gene expression.

\section{Abbreviations}

CESC: Cervical squamous cell carcinoma and endocervical adenocarcinoma; CIBERSORT: Cell type identification by estimating relative subsets of known RNA; DUOX1: Dual oxidase 1; DUOX2: Dual oxidase 2; ECL: Enhanced chemiluminescence; GEO: Gene Expression Omnibus; GEPIA: Gene Expression Profiling Interactive Analysis; GTEX: Genotype-Tissue Expression; HPA: Human Protein Atlas; IFN: Interferon; IHC: Immunohistochemical; JAKVSTAT: Janus kinase/signal transducers and activators of transcription; KEGG: Kyoto Encyclopedia of Genes and Genomes; NADPH: Nicotinamide adenine dinucleotide phosphate; NES: Normalized Enrichment Score; NK: Natural killer; NOX: Nicotinamide adenine dinucleotide phosphate oxidase; RIGI: Retinoic acid-inducible gene I; ROS: Reactive oxygen species; TCGA: The Cancer Genome Atlas

\section{Authors' contributions}

SYC, HSE, BSL, and SK conceived of the study. SYC, GK, and SK performed data analysis for experiments. SYC, MJS, and SK drafted the final version of the manuscript and figure legends. SYC, GK, PS, HJY, YBK, and SK revised the figures, added critical content to the discussion and were responsible in revising all portions of the submitted portion of the manuscript. $\mathrm{HC}$ and HNK performed western blot experiment using cervical cancer and control tissue. All contributors meet the criteria for authorship. All authors read and approved the final manuscript. 


\section{Funding}

This work was supported by a grant from Korea Institute of Oriental Medicine (K18123), National Research Foundation of Korea funded by the Korean government (NRF-2017R1C1B1004924) and Bio \& Medical Technology Development Program of the National Research Foundation (NRF) \& funded by the Korean government (NRF-2019M3E5D1A02068557). The funding bodies had no involvement in the design of the study, collection, analysis, and interpretation of data and in writing the manuscript.

\section{Availability of data and materials}

The data set is available in Gene Expression Omnibus (GEO) (https://www. ncbi.nlm.nih.gov/geo/) with the accession number: GSE75132.

\section{Ethics approval and consent to participate}

The results shown here are based upon data generated by the TCGA Research Network: http://cancergenome.nih.gov. All data downloaded from TCGA is publicly accessible and de-identified. Patients were consented by the TCGA Research Network. Documentation about consent and sample acquisition is publicly posted: https://cancergenome.nih.gov/abouttcga/policies. For human samples, all patients gave written informed consent for this study, which was approved by the Institutional Review Board of Chungnam National University Hospital (IRB number: 2019-05-087).

\section{Consent for publication}

Not applicable.

\section{Competing interests}

The authors declare that they have no competing interests.

\section{Author details}

${ }^{1}$ School of Medicine, Chungnam National University, 266 Munwha-ro, Jung-gu, Daejeon, Republic of Korea. ${ }^{2}$ Department of Clinical Medicine, Korea Institute of Oriental Medicine, 1672 Yuseong-daero, Yuseong-gu, Daejeon, Republic of Korea. ${ }^{3}$ Department of Anatomy, College of Medicine, Seoul National University, 103, Daehak-ro, Jongno-gu, Seoul, Republic of Korea. ${ }^{4}$ Department of Microbiology and Immunology, School of Medicine, Chonbuk National University, 20 Geonji-ro, Jeonju, Republic of Korea. ${ }^{5}$ Brain Korea 21 PLUS project for Medical Science, Chungnam National University, 266 Munwha-ro, Jung-gu, Daejeon, Republic of Korea. ${ }^{6}$ Research Institute of Medical Sciences, School of Medicine, Chungnam National University, 266 Munwha-ro, Jung-gu, Daejeon, Republic of Korea. ${ }^{7}$ Department of Obstetrics and Gynecology, Chungnam National University Hospital, 282 Munwha-ro, Jung-gu, Daejeon, Republic of Korea. ${ }^{8}$ Department of Obstetrics and Gynecology, School of Medicine, Chungnam National University, 266 Munwha-ro, Jung-gu, Daejeon, Republic of Korea. ${ }^{9}$ Department of Internal Medicine, Chungnam National University Hospital, 282 Munwha-ro, Jung-gu, Daejeon, Republic of Korea. ${ }^{10}$ Department of Internal Medicine, School of Medicine, Chungnam National University, 266 Munwha-ro, Jung-gu, Daejeon, Republic of Korea.

Received: 5 October 2018 Accepted: 24 September 2019

Published online: 09 November 2019

\section{References}

1. McCredie MR, Sharples KJ, Paul C, Baranyai J, Medley G, Jones RW, et al. Natural history of cervical neoplasia and risk of invasive cancer in women with cervical intraepithelial neoplasia 3: a retrospective cohort study. Lancet Oncol. 2008:9:425-34.

2. Cromme FV, Meijer CJ, Snijders PJ, Uyterlinde A, Kenemans P, Helmerhorst T, et al. Analysis of MHC class I and II expression in relation to presence of HPV genotypes in premalignant and malignant cervical lesions. $\mathrm{Br} J$ Cancer. 1993;67:1372-80.

3. Finn OJ. Immuno-oncology: understanding the function and dysfunction of the immune system in cancer. Ann Oncol. 2012;23:6-9.

4. Bedard K, Krause KH. The NOX family of ROS-generating NADPH oxidases: physiology and pathophysiology. Physiol Rev. 2007:87:245-313.

5. Finkel T, Holbrook NJ. Oxidants, oxidative stress and the biology of ageing Nature. 2000;408:239-47.

6. Roy K, Wu Y, Meitzler JL, Juhasz A, Liu H, Jiang G, et al. NADPH oxidases and cancer. Clin Sci (Lond). 2015;128:863-75.
7. Rada B, Leto TL. Oxidative innate immune defenses by Nox/Duox family NADPH oxidases. Contrib Microbiol. 2008;15:164-87.

8. Bae YS, Choi MK, Lee WJ. Dual oxidase in mucosal immunity and hostmicrobe homeostasis. Trends Immunol. 2010;31:278-87.

9. Geiszt M, Witta J, Baffi J, Lekstrom K, Leto TL. Dual oxidases represent novel hydrogen peroxide sources supporting mucosal surface host defense. FASEB J. 2003;17:1502-4

10. Edens WA, Sharling L, Cheng G, Shapira R, Kinkade JM, Lee T, et al. Tyrosine cross-linking of extracellular matrix is catalyzed by Duox, a multidomain oxidase/peroxidase with homology to the phagocyte oxidase subunit gp91phox. J Cell Biol. 2001;154:879-91.

11. Anh NT, Nishitani M, Harada S, Yamaguchi M, Kamei K. Essential role of Duox in stabilization of Drosophila wing. J Biol Chem. 2011;286:33244-51.

12. Xiao $X$, Yang L, Pang $X$, Zhang $R$, Zhu Y, Wang $P$, et al. A mesh-Duox pathway regulates homeostasis in the insect gut. Nat Microbiol. 2017;2: 17020.

13. Flores MV, Crawford KC, Pullin LM, Hall CJ, Crosier KE, Crosier PS. Dual oxidase in the intestinal epithelium of zebrafish larvae has anti-bacterial properties. Biochem Biophys Res Commun. 2010;400:164-8.

14. Kawahara T, Quinn MT, Lambeth JD. Molecular evolution of the reactive oxygen-generating NADPH oxidase (Nox/Duox) family of enzymes. BMC Evol Biol. 2007;7:109.

15. Kanehisa M, Goto S. KEGG: Kyoto encyclopedia of genes and genomes. Nucleic Acids Res. 2000;28:27-30.

16. Newman AM, Liu CL, Green MR, Gentles AJ, Feng W, Xu Y, et al. Robust enumeration of cell subsets from tissue expression profiles. Nat Methods. 2015:12:453-7.

17. Strengert M, Jennings $R$, Davanture $S$, Hayes $P$, Gabriel G, Knaus UG Mucosal reactive oxygen species are required for antiviral response: role of Duox in influenza a virus infection. Antioxid Redox Signal. 2014;20:2695-709.

18. Dupuy C, Ohayon R, Valent A, Noel-Hudson MS, Deme D, Virion A. Purification of a novel flavoprotein involved in the thyroid NADPH oxidase. Cloning of the porcine and human cDNAs. J Biol Chem. 1999;274:37265-9.

19. De Deken X, Wang D, Many MC, Costagliola S, Libert F, Vassart G, et al. Cloning of two human thyroid cDNAs encoding new members of the NADPH oxidase family. J Biol Chem. 2000;275:23227-33.

20. Degroot LJ, Niepomniszcze H. Biosynthesis of thyroid hormone: basic and clinical aspects. Metabolism. 1977;26:665-718.

21. Ameziane-El-Hassani R, Talbot M, de Souza Dos Santos MC, Al Ghuzlan A, Hartl D, Bidart JM, et al. NADPH oxidase DUOX1 promotes long-term persistence of oxidative stress after an exposure to irradiation. Proc Natl Acad Sci U S A. 2015;112:5051-6.

22. Ameziane-El-Hassani R, Schlumberger M, Dupuy C. NADPH oxidases: new actors in thyroid cancer? Nat Rev Endocrinol. 2016:12:485-94.

23. Little AC, Sulovari A, Danyal K, Heppner DE, Seward DJ, van der Vliet A. Paradoxical roles of dual oxidases in cancer biology. Free Radic Biol Med. 2017:110:117-32

24. Luxen S, Belinsky SA, Knaus UG. Silencing of DUOX NADPH oxidases by promoter hypermethylation in lung cancer. Cancer Res. 2008;68:1037-45.

25. Little AC, Sham D, Hristova M, Danyal K, Heppner DE, Bauer RA, et al. DUOX1 silencing in lung cancer promotes EMT, cancer stem cell characteristics and invasive properties. Oncogenesis. 2016;5:e261.

26. Aviello G, Knaus UG. ROS in gastrointestinal inflammation: rescue or sabotage? Br J Pharmacol. 2017;174:1704-18.

27. You X, Ma M, Hou G, Hu Y, Shi X. Gene expression and prognosis of NOX family members in gastric cancer. Onco Targets Ther. 2018;11:3065-74.

28. El Hassani RA, Benfares N, Caillou B, Talbot M, Sabourin JC, Belotte V, et al. Dual oxidase2 is expressed all along the digestive tract. Am J Physiol Gastrointest Liver Physiol. 2005;288:G933-42.

29. MacFie TS, Poulsom R, Parker A, Warnes G, Boitsova T, Nijhuis A, et al. DUOX2 and DUOXA2 form the predominant enzyme system capable of producing the reactive oxygen species $\mathrm{H}_{2} \mathrm{O} 2$ in active ulcerative colitis and are modulated by 5-aminosalicylic acid. Inflamm Bowel Dis. 2014;20:514-24.

30. Deep G, Kumar R, Jain AK, Dhar D, Panigrahi GK, Hussain A, et al. Graviola inhibits hypoxia-induced NADPH oxidase activity in prostate cancer cells reducing their proliferation and clonogenicity. Sci Rep. 2016;6:23135.

31. Guo Y, Han B, Luo K, Ren Z, Cai L, Sun L. NOX2-ROS-HIF-1alpha signaling is critical for the inhibitory effect of oleanolic acid on rectal cancer cell proliferation. Biomed Pharmacother. 2017;85:733-9.

32. Mukawera E, Chartier S, Williams V, Pagano PJ, Lapointe R, Grandvaux N. Redox-modulating agents target NOX2-dependent IKKepsilon oncogenic 
kinase expression and proliferation in human breast cancer cell lines. Redox Biol. 2015;6:9-18.

33. Parker BS, Rautela J, Hertzog PJ. Antitumour actions of interferons: implications for cancer therapy. Nat Rev Cancer. 2016;16:131-44.

34. Kwon J, Shatynski KE, Chen H, Morand S, de Deken X, Miot F, et al. The nonphagocytic NADPH oxidase Duox1 mediates a positive feedback loop during T cell receptor signaling. Sci Signal. 2010;3:ra59.

35. Rada B, Park JJ, Sil P, Geiszt M, Leto TL. NLRP3 inflammasome activation and interleukin-1 beta release in macrophages require calcium but are independent of calcium-activated NADPH oxidases. Inflamm Res. 2014;63: 821-30.

36. Habibovic A, Hristova M, Heppner DE, Danyal K, Ather JL, Janssen-Heininger YM, et al. DUOX1 mediates persistent epithelial EGFR activation, mucous cell metaplasia, and airway remodeling during allergic asthma. JCI Insight. 2016; 1:e88811.

37. Deffert C, Cachat J, Krause KH. Phagocyte NADPH oxidase, chronic granulomatous disease and mycobacterial infections. Cell Microbiol. 2014;16: 1168-78.

38. Fridman WH, Pages F, Sautes-Fridman C, Galon J. The immune contexture in human tumours: impact on clinical outcome. Nat Rev Cancer. 2012;12: 298-306.

39. Langers I, Renoux V, Reschner A, Touze A, Coursaget P, Boniver J, et al. Natural killer and dendritic cells collaborate in the immune response induced by the vaccine against uterine cervical cancer. Eur I Immunol. 2014;44:3585-95.

40. Palucka K, Banchereau J. Cancer immunotherapy via dendritic cells. Nat Rev Cancer. 2012;12:265-77.

41. Knutson KL, Disis ML. Tumor antigen-specific Thelper cells in cancer immunity and immunotherapy. Cancer Immunol Immunother. 2005;54:721-8.

42. Marichal T, Tsai M, Galli SJ. Mast cells: potential positive and negative roles in tumor biology. Cancer Immunol Res. 2013;1:269-79.

\section{Publisher's Note}

Springer Nature remains neutral with regard to jurisdictional claims in published maps and institutional affiliations.

Ready to submit your research? Choose BMC and benefit from:

- fast, convenient online submission

- thorough peer review by experienced researchers in your field

- rapid publication on acceptance

- support for research data, including large and complex data types

- gold Open Access which fosters wider collaboration and increased citations

- maximum visibility for your research: over $100 \mathrm{M}$ website views per year

At $\mathrm{BMC}$, research is always in progress.

Learn more biomedcentral.com/submissions 